\title{
Heterogeneous HIV-1 Reactivation Patterns of Disulfiram and Combined Disulfiram+Romidepsin Treatments
}

\author{
Anna Kula, PhD,,$^{a, b}$ Nadège Delacourt, MSc, ${ }^{a}$ Sophie Bouchat, PhD, ${ }^{a}$ Gilles Darcis, MD, PhD, ${ }^{a, c, d}$ \\ Veronique Avettand-Fenoel, PhD, ${ }^{e, f}$ Roxane Verdikt, PhD, ${ }^{a}$ Francis Corazza, MD, PhD, ${ }^{g}$ \\ Coca Necsoi, MD, PhD, ${ }^{h}$ Caroline Vanhulle, MSc, ${ }^{a}$ Maryam Bendoumou, MSc, ${ }^{a}$ Arsene Burny, ${ }^{a}$ \\ Stephane De Wit, MD, PhD, ${ }^{h}$ Christine Rouzioux, ${ }^{e, f}$ Oliver Rohr, ${ }^{i, j}$ and Carine Van Lint ${ }^{a}$
}

Objectives: Few single latency-reversing agents (LRAs) have been tested in vivo, and only some of them have demonstrated an effect, albeit weak, on the decrease of latent reservoir. Therefore, other LRAs and combinations of LRAs need to be assessed. Here, we evaluated the potential of combined treatments of therapeutically promising LRAs, disulfiram and romidepsin.

Received for publication June 28, 2018; accepted December 3, 2018.

From the a Service of Molecular Virology, Institut de Biologie et de Médecine Moléculaires (IBMM), Université Libre de Bruxelles (ULB), Gosselies, Belgium; ${ }^{b}$ Malopolska Centre of Biotechnology, Laboratory of Virology, Jagiellonian University, Krakow, Poland; ${ }^{\mathrm{C} A c a d e m i c ~ M e d i c a l ~ C e n t e r ~ o f ~ t h e ~}$ University of Amsterdam, Amsterdam, The Netherlands; ${ }^{\mathrm{d}}$ Infectious Diseases Department, Liege University Hospital, Liege, Belgium; 'Service de Virologie, Université Paris-Descartes, AP-HP, Hopital Necker-Enfants Malades, Paris, France; ${ }^{\mathrm{f} U n i v e r s i t e ́ ~ P a r i s ~ d e s c a r t e s, ~ S o r b o n n e ~ P a r i s ~ C i t e ́, ~}$ Paris, France; ${ }^{g}$ Laboratory of Immunology, Brugmann University Hospital, Université Libre de Bruxelles (ULB), Bruxelles, Belgium; ' hervice des Maladies Infectieuses, CHU St-Pierre, ULB, Bruxelles, Belgium; institut de Parasitologie et de Pathologie Tropicale, Strasbourg, France; and ${ }^{\mathrm{j}}$ Institut Universitaire de Technologie Louis Pasteur de Schiltigheim, University of Strasbourg, Strasbourg, France.

AQ:3

This project has received funding from the European Union's Horizon 2020 research and innovation programme under grant agreement No 691119EU4HIVCURE-H2020-MSCA-RISE-2015 to C.V.L. and under the National Science Centre (Poland) and Marie Skłodowska-Curie grant agreement no. 665778, Polonez Grant UMO-2015/19/P/NZ6/02188 to A.K. Work in C.V.L.'s laboratory was supported from the Belgian Fund for Scientific Research (FRS-FNRS, Belgium), the "Fondation Roi Baudouin", the NEAT program (Networking to Enhance the Use of Economics in Animal Health Education, Research, and Policy Making), the Walloon Region (Fonds de Maturation), "Les Amis des Instituts Pasteur à Bruxelles, asbl, and the University of Brussels (Action de Recherche Concertée (ARC) grant). The laboratory of C.V.L. is part of the ULB-Cancer Research Centre (U-CRC). A.K. is a postdoctoral fellow of "Les Amis des Instituts Pasteur à Bruxelles, asbl. C.V.L. is "Directeur de Recherches" of the FRS-FNRS (Belgium), respectively.

The authors declare no competing financial interests.

AQ:4 The authors S.B. and G.D. have equally contributed to the article.

Supplemental digital content is available for this article. Direct URL citations appear in the printed text and are provided in the HTML and PDF versions of this article on the journal's Web site (www.jaids.com).

Correspondence to: Carine Van Lint, Service of Molecular Virology, Institut de Biologie et de Médecine Moléculaires (IBMM), Université Libre de Bruxelles (ULB), Rue des Professeurs Jeener et Brachet 12, 6041 Gosselies, Belgium (e-mail: cvlint@ulb.ac.be).

Copyright (c) 2019 The Author(s). Published by Wolters Kluwer Health, Inc. This is an open-access article distributed under the terms of the Creative Commons Attribution-Non Commercial-No Derivatives License 4.0 (CCBY-NC-ND), where it is permissible to download and share the work provided it is properly cited. The work cannot be changed in any way or used commercially without permission from the journal.
Setting and Methods: We assessed the reactivation potential of individual disulfiram or simultaneous or sequential combined treatments with romidepsin in vitro in latently infected cell lines of T-lymphoid and myeloid origins and in ex vivo cultures of $\mathrm{CD}^{+}-$ depleted peripheral blood mononuclear cells isolated from $18 \mathrm{HIV}_{-1}{ }^{+}$ combination antiretroviral therapy-treated individuals.

Results: We demonstrated heterogeneous reactivation effects of disulfiram in vitro in various cell lines of myeloid origin and no latency reversal neither in vitro in T-lymphoid cells nor ex vivo, even if doses corresponding to maximal plasmatic concentration or higher were tested. Disulfiram+romidepsin combined treatments produced distinct reactivation patterns in vitro. Ex vivo, the combined treatments showed a modest reactivation effect when used simultaneously as opposed to no viral reactivation for the corresponding sequential treatment.

Conclusions: Exclusive reactivation effects of disulfiram in myeloid latency cell lines suggest that disulfiram could be a potential LRA for this neglected reservoir. Moreover, distinct reactivation profiles pinpoint heterogeneity of the latent reservoir and confirm that the mechanisms that contribute to HIV latency are diverse. Importantly, disulfiram+romidepsin treatments are not potent ex vivo and most likely do not represent an effective drug combination to achieve high levels of latency reversal in vivo.

Key Words: disulfiram, romidepsin, latency-reversing agents, heterogeneity of HIV reservoir

(J Acquir Immune Defic Syndr 2019;00:1-9)

\section{INTRODUCTION}

Combination antiretroviral therapy (cART) has profound health benefits for people with HIV, but cART is not curative and patients must stay on therapy indefinitely. Persistence during cART of latently infected cells containing integrated, transcriptionally silent but replication-competent proviruses is a major hurdle for HIV-1 eradication. ${ }^{1}$ Although many cells may contribute to the latent reservoirs, including monocytes and monocyte-derived macrophages (reviewed in Refs. 2,3), the best characterized one is a small population of long-lived HIV-1-infected resting memory $\mathrm{CD}^{+} \mathrm{T}$ cells, maintained throughout patient's life by homeostatic proliferation. ${ }^{4}$ The absence of viral gene expression in latently infected cells renders the virus imperceptible to host immune 
response. However, various cellular stimuli can induce the latent reservoirs, allowing for HIV-1 transcription and subsequent production of replication-competent virus, ${ }^{5,6}$ which can represent one potential source of rebound of viremia after cART interruption. ${ }^{7}$ One proposed strategy for eliminating the latent reservoir is to pharmacologically stimulate HIV-1 gene expression in latently infected cells, rendering these cells susceptible to cytolytic T lymphocytes or viral cytopathic effects while maintaining cART to prevent new spreading infection. ${ }^{8}$ Small molecule latency-reversing agents (LRAs) with potential therapeutical application as shock agents have been identified including antialcoholism drug disulfiram (DSF) ${ }^{9,10}$ and histone deacetylase inhibitors (HDACIs) such as SAHA, panobinostat, and romidepsin. ${ }^{11-13}$ Such compounds also include protein kinase $\mathrm{C}$ agonists (prostratin, bryostatin, and ingenols), ${ }^{14,15}$ bromodomain and extraterminal domain inhibitors (JQ1, I-BET, I-and BET151), ${ }^{6}$ histone methyltransferases inhibitors (chaetocin and BIX0194), ${ }^{16}$ DNA methyltransferase inhibitors or demethylating agents $(5-A z a d C),{ }^{5}$ and toll-like receptor 7 agonists (GS-9620). ${ }^{17}$ DSF has been used for the treatment of chronic alcoholism for more than 60 years. ${ }^{18}$ In vitro studies showed that DSF reactivates HIV in latently infected cell lines of myeloid but not T-lymphoid origin ${ }^{19}$ and in primary Bcl-2-transduced $\mathrm{CD}^{+}$T-cell model of latency. ${ }^{20}$ A pilot clinical study using an FDA-approved dose showed no overall effect on plasma HIV RNA during DSF administration, but a transient increase in plasma HIV RNA was noted in a post hoc analysis in some patients. ${ }^{9}$ Importantly, a dose-escalation study showed very good safety profile of DSF, a clear dose-dependent effect of disulfiram on HIV transcription; however, a small effect on plasma HIV-1 RNA only for the highest dose was observed. ${ }^{10}$ In addition to antilatency clinical trials using DSF, several trials using HDACIs have been reported in the HIV field. ${ }^{12,13}$ Romidepsin, an HDACI, was also evaluated in vivo and was shown to be the most potent latency activator tested in clinical trials to date. ${ }^{13}$ However, despite its potent effect, no decrease in the reservoir size was observed. Altogether, these studies using LRAs are encouraging but question the efficacy of LRAs used alone to reduce the size of the HIV-1 reservoirs. Combination of different classes of LRAs targeting different mechanisms of latency can exhibit synergistic effect (ie, result in a higher reactivation level than the sum of the reactivations produced by each compound individually) in reactivation of HIV expression in latently infected cells. We and others have demonstrated proof of concepts for the coadministration of 2 different classes of LRAs ${ }^{5,6,21}$ as a therapeutic perspective to decrease the pool of latent HIV-1 reservoirs in the presence of efficient cART.

In this study, we turned our attention to 2 therapeutically promising LRAs, DSF and romidepsin, since the former showed excellent safety profile and the latter potent reactivation in vivo. We evaluated the reactivation potential of individual and combined treatments of DSF and romidepsin in multiple in vitro cellular latency models and in ex vivo cultures of $\mathrm{CD}^{+}$-depleted peripheral blood mononuclear cells (PBMCs) isolated from $\mathrm{HIV}^{+}$aviremic cART-treated individuals.

\section{RESULTS}

\section{Heterogeneous Effects of DSF on HIV Reactivation In Vitro in Latently Infected Cell Lines}

We first determined optimal concentration of DSF in terms of both HIV-1 reactivation potential and effect on the cellular viability. We measured induction of HIV-1 p24 capsid protein production and cellular metabolic activity in wellstudied HIV-1 latency cellular model, promonocytic U1 cell line. Increasing doses of DSF for 24, 48, and 72 hours augmented p24 antigen level in the cell supernatants in a doseand time-dependent manner from 5 to $50 \mu \mathrm{M}$ (Fig. 1A). We also assessed the effect of the increasing doses of DSF on cell viability as measured by WST-1 assay that reflects the cell metabolic activity and cell proliferation. We observed doseand time-dependent effect on cellular viability for DSF doses ranging from 20 to $50 \mu \mathrm{M}$ (Fig. 1B). We therefore determined that $10 \mu \mathrm{M}$ of DSF for 48 hours was optimal in terms of both reactivation effect and effect on cellular viability.

We next assessed the reactivation effect of increasing doses of DSF in 2 other monocytic-derived cell lines containing latent HIV-1 proviruses, that is, THP89GF and CHME-5/HIV microglial cells. These cell lines containing a reporter GFP were treated with increasing doses of DSF for 24, 48, and 72 hours (Figs. 1C, E, respectively). In THP89GFP cells, $20 \mu \mathrm{M}$ of DSF for 24 hours was optimal in terms of both reactivation effect and effect on cellular viability. Interestingly, in the CHME-5/HIV cell line, we observed 2 peaks of reactivation (at 0.5 and $10 \mu \mathrm{M}$ ), and the reactivation effect was the most pronounced after 24-hour stimulation with strong decrease in reactivation after 48 and 72 hours of DSF treatment (Fig. 1E). Moreover, cytotoxicity was observed from 20- $\mu$ M DSF (Fig. 1F).

Finally, we also assessed the reactivation effect of increasing doses of DSF in a T-lymphoid Jurkat cell-based latency model, the J-Lat 9.2 cell line treated for 24,48 , and 72 hours (Fig. 1G). We observed no induction of HIV-1 gene expression in this cell line at any DSF concentration used and at any time-point tested as compared to TNFalpha treatment (see Figure 1, Supplemental Digital Content, http://links.lww. com/QAI/B273). DSF presented no negative effect on cellular viability for concentrations of DSF up to $20 \mu \mathrm{M}$ treated for 24 and 48 hours (Fig. 1H). In addition, no HIV reactivation could be observed in 2 other Jurkat-based latency model cell lines, that is, A2 and A72 cells (see Figure 2, Supplemental Digital Content, http://links.lww.com/QAI/B273). Importantly, Doyon et $\mathrm{al}^{19}$ has also reported that DSF reactivates HIV-1 expression in the U1 but not in Jurkat-based cell line latency models. They have shown that DSF significantly reduces phosphatase and tensin homolog (PTEN) protein levels in U1 cells. They have observed that Jurkat cell-based models of latency do not express PTEN, explaining the lack of DSF effect on HIV-1 expression in these cell lines. Notably, we could not observe any expression levels of PTEN protein neither in J-Lat 9.2 nor in monocytic-derived U1 and THP89GFP cells (see Figure 3, Supplemental Digital Content, http://links.lww.com/QAI/B273), but we detected PTEN in CHME-5/HIV microglial cell line. 

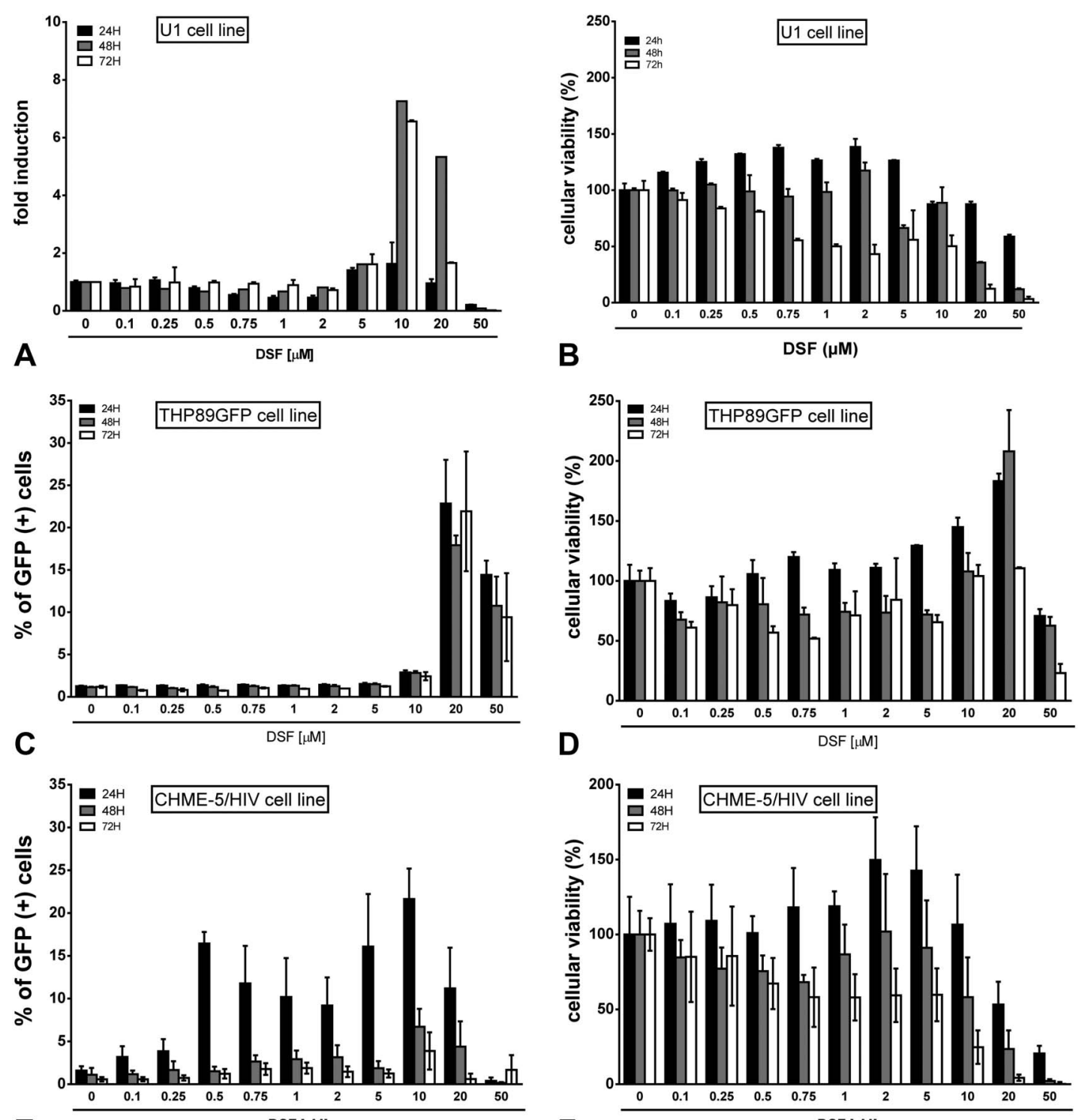

E

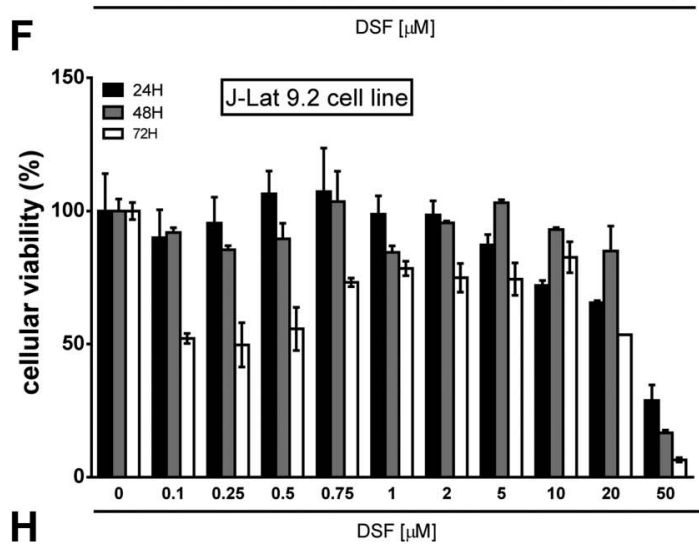

FIGURE 1. Dose- and time-dependent effect of DSF on HIV-1 production and cellular viability in vitro. Cells were mock-treated or treated with increasing doses of DSF as indicated. At 24, 48, and 72 hours after treatment, either CA-p24 ELISA production in cell supernatants (A) was measured or viral protein expression was analyzed by FACS ( $C$, $E$, and $G$ ). In addition, cellular viability was assessed by WST-1 assay that reflects cell metabolic activity, proliferation, and cytotoxicity $(B, D, F$, and $H)$. Results with the mock-treated cells were arbitrary set at a value of $1(A)$ or $1 \%$ of GFP (+) cells $(C, E$, and $G)$ or $100 \%(B, D, F$, and $H)$. Mean values and SEs of the mean values from duplicate samples are indicated. One representative experiment from 3 is represented. 
In conclusion, our results demonstrated that the in vitro reactivation effect of DSF was heterogeneous with distinct reactivation patterns within myeloid-derived latency cell lines and no reactivation was observed in T-lymphocytic-based latency cell lines.

\section{Distinct In Vitro Reactivation Patterns After DSF+Romidepsin Combined Treatments in Myeloid HIV Latently Infected Cell Lines}

We next investigated whether DSF could synergistically reactivate HIV-1 expression when combined with romidepsin in either U1 or THP89GFP or CHME-5/HIV cell lines. Two compounds synergize when their combination produces higher effect than the sum of effects arising from separate treatments. We tested DSF at $0.5,10$, and $20 \mu \mathrm{M}$ (concentrations we determined to be optimal doses in latency models we investigated). In addition, we also investigated DSF at 2 and $5 \mu \mathrm{M}$. Romidepsin was tested at 2 concentrations 0.0175 and $0.04 \mu \mathrm{M}$ that correspond to dose previously established by our laboratory ${ }^{5}$ and to plasmatic concentration achieved in patients after romidepsin administration, ${ }^{13}$ respectively.

Since, in U1 cells, we observed time-dependent effect of DSF with optimal timing at 48 hours, we investigated both simultaneous and sequential DSF+romidepsin combined treatments. We compared a simultaneous treatment in which DSF and romidepsin were added together for 48 hours with a sequential treatment in which 24-hour DSF pretreatment was followed by a 24-hour romidepsin induction, corresponding to a total of 48-hour DSF treatment. By extracellular p24 ELISA assays, we observed that simultaneous DSF+romidepsin treatments exhibited overall only additive effects (Fig. 2A, max. fold synergy $=1.1$ ) as compared to sequential treatment that produced synergistic effects (Fig. 2B, max. fold synergy
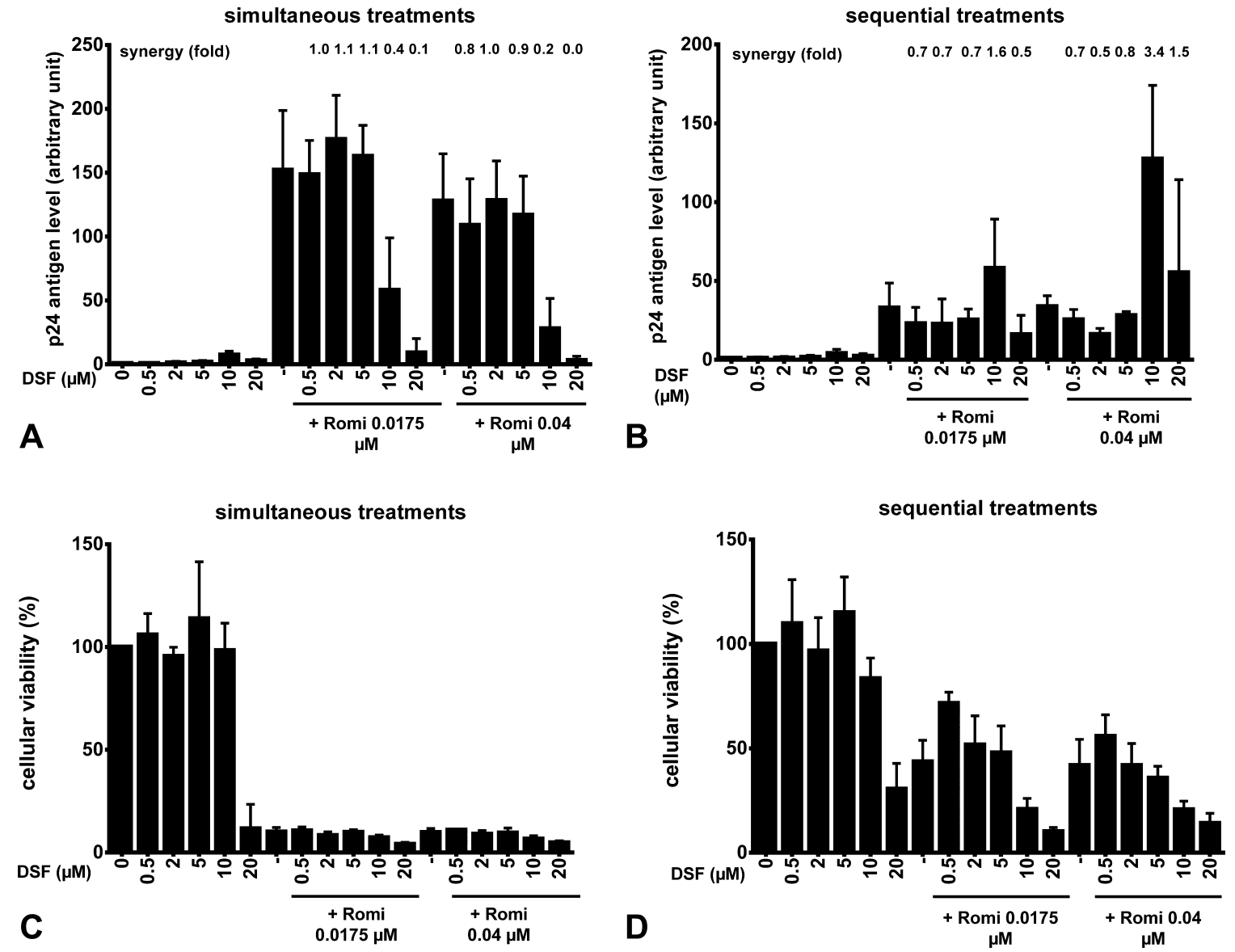

AQ:6 FIGURE 2. Comparison of simultaneous DSF+romidepsin combined treatments with its corresponding sequential treatments in U1 cells. Cells were mock-treated or treated with DSF or romidepsin alone or in combinations as indicated. Regarding sequential treatment, at 24 hours after romidepsin treatment and 48 hours after DSF treatment, viral production was estimated by measuring CA-p24 antigen concentration in culture supernatants. Regarding simultaneous treatment, at 48 hours after DSF and romidepsin treatments, viral production was estimated by measuring CA-p24 antigen concentration in culture supernatants. The mocktreated value was arbitrarily set at a value of 1 . Mean values and SEs of the mean values from triplicate samples are indicated. In addition, cellular viability was assessed by WST-1 assay that reflects cell metabolic activity, proliferation, and cytotoxicity. For each combinatory treatment, the fold synergy was calculated by dividing the effect observed after cotreatments by the sum of the effects obtained after the individual treatments. 
$=3.4)$. Of note, 48-hour romidepsin treatment was very toxic (90\% of reduction in cellular viability) as compared to $24-$ hour romidepsin treatment $(60 \%$ of reduction in cellular viability) (Figs. 2C, D, respectively). Worth mentioning, we observed very high synergistic reactivation effects of sequential treatments in which DSF was combined with some other HDACIs (see Figure 4A, Supplemental Digital Content, http://links.lww.com/QAI/B273) and other classes of LRAs (see Figure 4B, Supplemental Digital Content, http://links. lww.com/QAI/B273).

Next, we evaluated whether DSF could synergize with romidepsin in THP89GFP and CHME-5/HIV cell lines. We investigated only the simultaneous combined treatments in these cell lines, since DSF reactivation effect was only observed after 24 hours of treatment. Regarding THP89GFP cells, we observed synergistic increase in the GFP-positive cells after simultaneous cotreatments of $20-\mu \mathrm{M}$ DSF with either romidepsin at $0.0175 \mu \mathrm{M}$ or at $0.04 \mu \mathrm{M}$ (Fig. 3A, fold synergy $=2.1$ and fold synergy $=2.3$, respectively). Marginal effects on cellular viability were observed (Fig. 3B). In case of CHME-5/

AQ:5 HIV cells, we observed only modest synergistic increase in the GFP-positive cells after simultaneous DSF+romidepsin combined treatments as compared to individual treatments (Fig. 3C, fold synergy $<2$, see Table S1, Supplemental Digital Content, http://links.lww.com/QAI/B273). Cytotoxicity was observed for $20-\mu \mathrm{M}$ DSF and the DSF+romidepsin combined treatments (Fig. 3D).

In conclusion, our results demonstrated that DSF+romidepsin combined treatments produced various synergistic effects, and the highest synergistic effects were observed in U1 cells after sequential DSF+romidepsin treatment.

\section{Simultaneous But Not Sequential DSF+Romidepsin Treatments Lead to Moderately Higher HIV-1 Recovery Than the Individual Drug Treatments in CD8+-Depleted PBMCs From cART-Treated HIV ${ }^{+}$ Aviremic Individuals}

To address the reactivation potential of the simultaneous and sequential DSF+romidepsin treatments in ex vivo cultures, we firstly evaluated the cytotoxic effects of those treatments on cells from healthy donors. We observed that

\section{THP89 cell line}

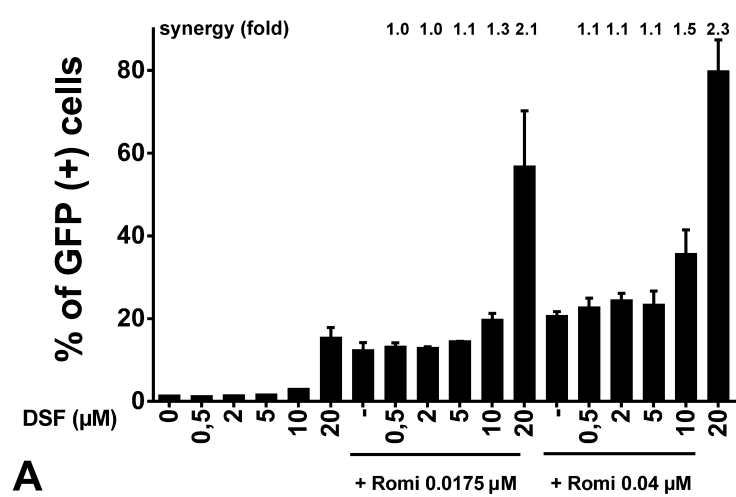

CHME-5/HIV cell line

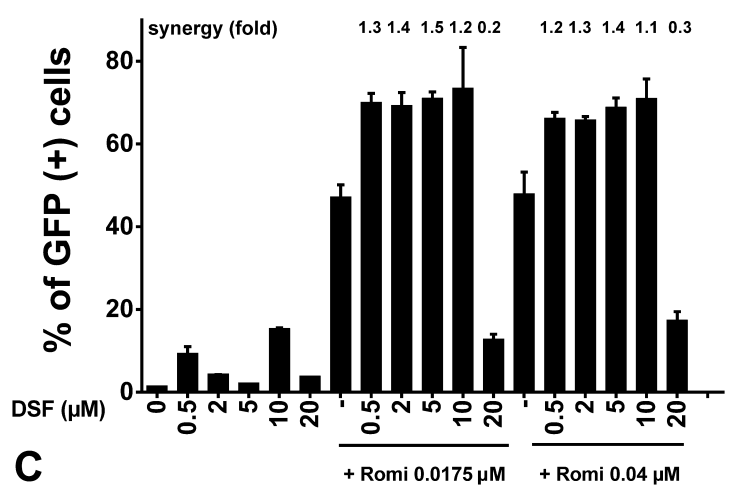

THP89 cell line

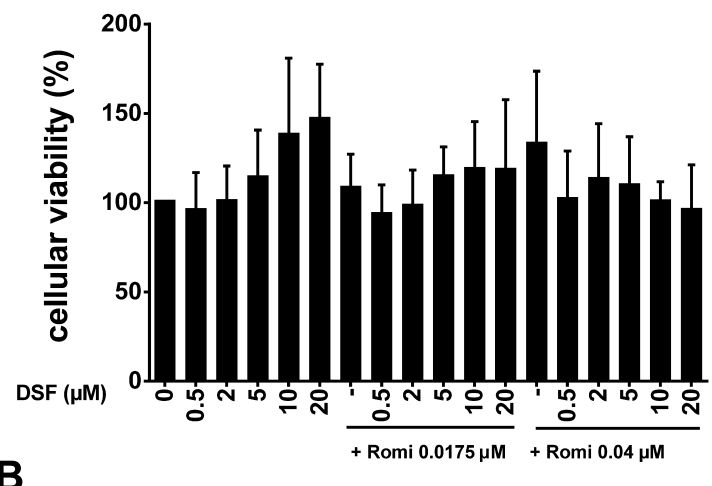

CHME-5/HIV cell line

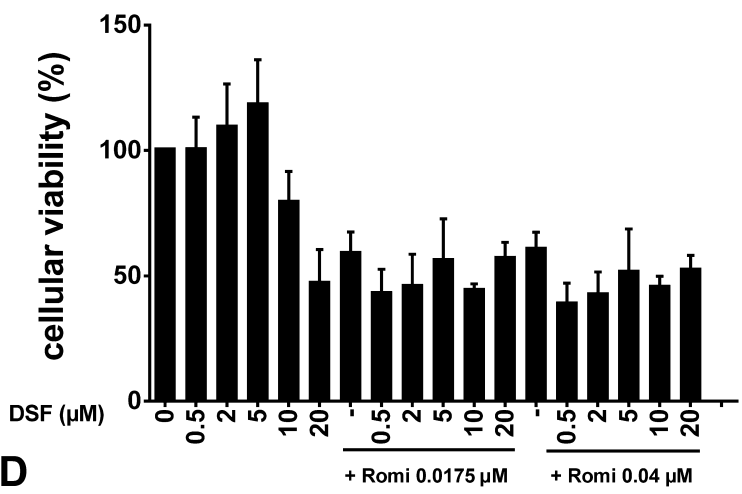

FIGURE 3. DSF+romidepsin simultaneous treatments moderately induce HIV-1 as compared to drugs alone in THP89GFP and CHME-5/HIV cell lines. The CHME-5/HIV microglial cells were mock-treated, treated with DSF, or romidepsin alone or in combination as indicated. At 24 hours after treatment, cells were analyzed by flow cytometry to quantify the proportion of cells expressing GFP. Mean values and SEs of the mean values from duplicate samples are indicated. One representative experiment from 3 is represented. 
DSF alone was not toxic and the combined treatments produced cytotoxic effects ranging from $20 \%$ to $60 \%$ as assessed by cellular viability tests (see Figure 5, Supplemental Digital Content, http://links.lww.com/QAI/B273). Then, $\mathrm{CD}^{+}$-depleted PBMCs isolated from 18 cART-treated aviremic $\mathrm{HIV}-1^{+}$patients were mock-treated, treated with antiCD3+anti-CD28 antibodies as a positive control for global T-cell activation or with indicated compounds added in a simultaneous or sequential manner alone or in combination

F4 (Fig. 4 and Table S1, Supplemental Digital Content, http:// links.lww.com/QAI/B273). HIV-1 genomic RNA concentrations in culture supernatants were quantified after 6 days of T1 $\operatorname{drug}(\mathrm{s})$ treatment (Table 1). We observed in mock-treated cultures a recovery in viral genomic RNA (mean of 928 HIV-1 RNA copies $/ \mathrm{mL}$ ) that could be explained by activation of HIV-infected cells during the purification procedure or during the course of experiment. Importantly, we observed that DSF at $2 \mu \mathrm{M}$ (that corresponds to plasmatic concentration reported by Elliot et $\mathrm{al}^{10}$ ) and DSF at $5 \mu \mathrm{M}$ (that has been chosen by us in this ex vivo study) showed no additional increase in mean HIV-1 RNA levels as compared to mocktreated cells (597 HIV-1 RNA copies/ml and 646 HIV-1 RNA copies/mL, respectively). However, these changes were not statistically significant (see Table S2, Supplemental Digital Content, http://links.lww.com/QAI/B273). Furthermore, 2 doses of romidepsin were selected for the ex vivo study: a dose of $0.0175 \mu \mathrm{M}$ that was determined in ${ }^{5}$ and a dose of $0.04 \mu \mathrm{M}$ that corresponds to plasmatic concentration measured in HIV reactivation clinical trial. ${ }^{13}$ Romidepsin at 0.0175 and $0.04 \mu \mathrm{M}$ that was added at day 1 exhibited statistically significant increases in HIV recovery (mean of 1925 HIV-1 RNA copies/mL and 2546 HIV-1 RNA copies/ $\mathrm{mL}$, respectively, Fig. 4, see Table S2, Supplemental Digital Content, http://links.lww.com/QAI/B273). Romidepsin at 0.0175 and $0.04 \mu \mathrm{M}$ that was added at day 3 produced lower
HIV recovery (mean of $1275 \mathrm{HIV}-1$ RNA copies/mL and 1617 HIV-1 RNA copies/mL, respectively, Fig. 4) that was not statistically relevant (see Table S2, Supplemental Digital Content, http://links.lww.com/QAI/B273). Combined simultaneous DSF+romidepsin treatments exhibited increases in extracellular HIV-1 RNA levels that were higher than their corresponding individual treatments (Fig. 4). Those increases were statistically significant when compared either with mock or individual DSF treatments, but no significance could be observed for comparisons with corresponding individual romidepsin treatment (see Table S2, Supplemental Digital Content, http://links.lww.com/QAI/B273). Of note, simultaneous combination of $5-\mu \mathrm{M}$ DSF and $0.04-\mu \mathrm{M}$ romidepsin induced the highest mean HIV RNA levels; however, this recovery was not statistically relevant when compared with romidepsin individual treatment (Fig. 4 and Table S2, Supplemental Digital Content, http://links.lww.com/QAI/ B273).

Moreover, sequential DSF+romidepsin treatments did not exhibit any positive and statistically relevant effects on extracellular HIV-1 RNA levels (Fig. 4 and Table S2, Supplemental Digital Content, http://links.lww.com/QAI/ B273).

In addition to the increases in mean extracellular HIV-1 RNA levels, we demonstrated a higher viral production in several cell cultures after combined treatments (Table 1, see italicized and bold values).

In conclusion, our results demonstrated that individual DSF and sequential DSF+romidepsin treatments did not reactivate HIV from latency ex vivo in $\mathrm{CD}^{+}$-depleted PBMCs from $\mathrm{HIV}^{+}$cART-treated aviremic individuals. Importantly, individual romidepsin treatments produced statistically significant HIV recoveries. Moreover, simultaneous DSF+romidepsin treatments produced only moderate beneficial reactivation effects.
FIGURE 4. Simultaneous but not sequential DSF+romidepsin treatments AQ:7 lead to moderately higher HIV-1 recovery than the drugs alone in $\mathrm{CD}^{+}$-depleted PBMCs from CART-treated $\mathrm{HIV}^{+}$aviremic patients. Ex vivo cultures of $\mathrm{CD}^{+}{ }_{-}$ depleted PBMCs purified from blood of 18 patients were mock-treated, treated with anti-CD3+anti-CD28 antibodies $(\mathrm{C}+), 2$ doses of DSF, and 2 doses of romidepsin alone or in combination as indicated. Six days after treatment, concentrations of extracellular viral RNA (EC HIV-1 RNA) in culture supernatants were determined. The results were reported as the actual HIV RNA copy numbers/ml or as an estimated value calculated as $50 \%$ of the smallest value when HIV RNA was not detected to assign a log value. Dashed line indicates the 300 HIV-1 RNA copies/ml limit of detection. The mean values are represented.

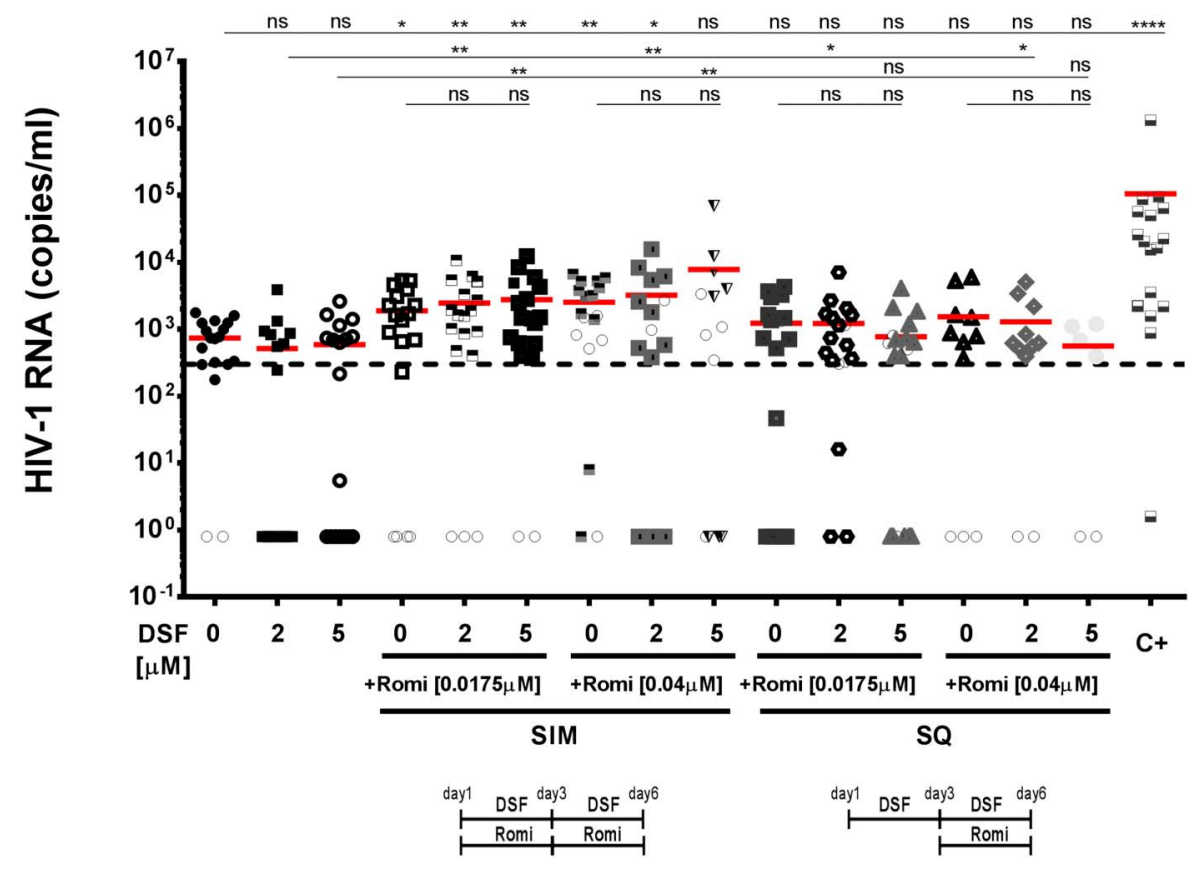

Copyright (C) 2019 The Author(s). Published by Wolters Kluwer Health, Inc. 
TABLE 1. Simultaneous and Sequential DSF+Romidepsin Treatments in CD8+-Depleted PBMCs From cART-Treated HIV ${ }^{+}$Aviremic Patients Simultaneous Sequential

LOG Total HIV-1

DNA (Copies $110^{6}$

Romi

(Copies $10^{6} \mathrm{CDB}^{+}-\mathrm{CDB}^{+}-\mathrm{Deps} / \mathrm{p}$

$0.0175 \mu \mathrm{M}+$

Romi

$0.0175 \mu \mathrm{M}+$

Romi 0.04

$\mu \mathrm{M}+$

Patient Depleted PBMCs)

PBMCs)

DSF DSF Romi

DSF Romi

DSF

Romi

DSF DSF Romi DSF DSF

\begin{tabular}{|c|c|c|c|c|c|c|c|}
\hline Patient & Denleted PBMCs) & PBMCs) & Mock & \multicolumn{3}{|c|}{$5.4 \mathrm{M}$} & $5 \mu \mathbf{M}$ \\
\hline
\end{tabular}

1567

$\begin{array}{lcccc}3.20 & 1601 & 3884 & \text { I } & 1633 \\ 2.90 & \text { I } & \text { I } & \text { I } & 5386 \\ 2.56 & 1337 & \text { I } & \text { I } & 1705\end{array}$

$\begin{array}{rrr}856 & 1479 & 817\end{array}$

NT NT I

I $1173 \quad$ NT

NT $\quad$ NT 2231

$\begin{array}{rrrrrrrrrrrr}10,690 & 4302 & 3798 & 8320 & \mathrm{I} & 3262 & 582 & 616 & 780 & \mathrm{I} & 1090 & 16,310 \\ 6172 & 2469 & 5281 & \mathrm{NT} & \mathrm{NT} & 4301 & 365 & \mathrm{I} & \mathrm{NT} & \mathrm{NT} & \mathrm{NT} & 95,290\end{array}$

$\begin{array}{ll}\mathrm{I} & \mathrm{I} \\ 8320 & \mathrm{I}\end{array}$

I

$\begin{array}{llllll}1141 & \mathbf{8 1 0} & \text { NT } & \text { NT } & \text { NT } & 15,320\end{array}$

$\begin{array}{lllllllllllllllll}2.50 & 1247 & 867 & 1141 & 1367 & 1945 & 398 & 1713 & \text { NT } & \text { NT } & 735 & 736 & 392 & \text { NT } & \text { NT } & \text { NT } & 86,990\end{array}$

$\begin{array}{rrrrrrrrrrrrrrrrr}2.49 & 720 & 1321 & \mathrm{I} & \mathrm{I} & 929 & 610 & 685 & 524 & 817 & \mathrm{I} & 1615 & \mathrm{I} & \mathrm{I} & \mathrm{I} & \mathrm{NT} & 879\end{array}$

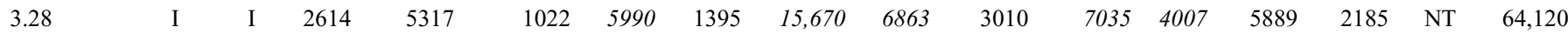

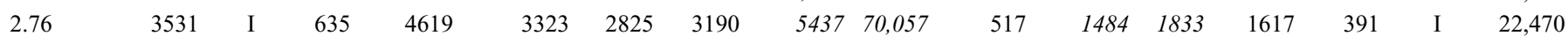

$\begin{array}{lllllllllllllllll}2.64 & 936 & \text { I } & \text { I } & 2280 & 1627 & 757 & 4166 & 380 & 4013 & 1353 & 2699 & 393 & 362 & 505 & 1187 & 25,850\end{array}$

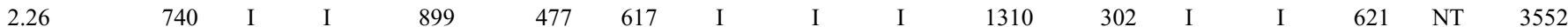

$\begin{array}{rllllllllllllllll}2.77 & 524 & 553 & 690 & 655 & \mathrm{I} & \mathrm{I} & \mathrm{I} & 580 & 346 & 1635 & 1689 & \mathrm{I} & \mathrm{I} & 5060 & \mathrm{I} & \mathrm{NT}\end{array}$

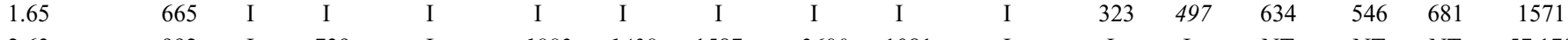

$\begin{array}{lllllllllllllllll}2.63 & 992 & \text { I } & 739 & \text { I } & 1882 & 1438 & 1587 & 2690 & 1081 & \text { I } & \text { I } & \text { I } & \text { NT } & \text { NT } & \text { NT } & 57,170\end{array}$

$\begin{array}{lllllllllllllllll}3.12 & 319 & 602 & 775 & 2282 & 2725 & 12,300 & 5917 & 2580 & 3072 & 3689 & 2054 & 730 & 866 & 843 & 392 & 1,326,000\end{array}$

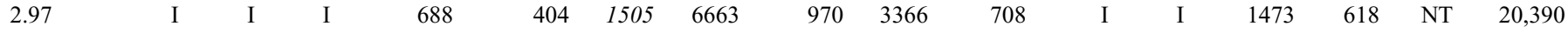

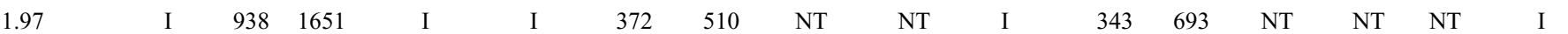

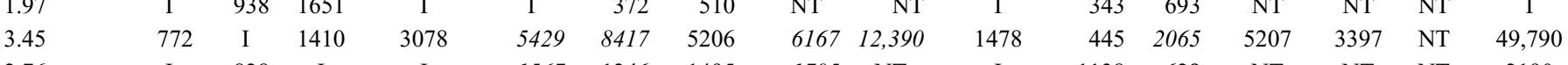

$\begin{array}{rrrcccccccccccccccccc}\text { P17 } & 2788 & 3.45 & 772 & \text { I } & 1410 & 3078 & 5429 & 8417 & 5206 & 6167 & 12,390 & 1478 & 445 & 2065 & 5207 & 3397 & \text { NT } & 49,790 \\ \text { P18 } & 570 & 2.76 & \text { I } & 828 & \text { I } & \text { I } & 1567 & \mathbf{1 2 4 6} & 1495 & 1795 & \text { NT } & \text { I } & 1138 & \mathbf{6 2 9} & \text { NT } & \text { NT } & \text { NT } & 2190\end{array}$

Cultures of CD8 ${ }^{+}$-depleted PBMCs purified from blood of 18 patients were mock-treated or treated with anti-CD3 ${ }^{+}$anti-CD28 antibodies $(\mathrm{C}+)$, DSF $(2$ and $5 \mu \mathrm{M})$ and romidepsin $(0.0175,0.04$ and $0.1 \mu \mathrm{M})$ alone, or in combination as indicated. Six days after treatment, concentrations of genomic viral RNA in culture supernatants were determined, and the values were expressed as HIV-1 RNA copies/ml. Total HIV-1 DNA was measured in cellular culture and was expressed as HIV-1 DNA copies $/ 10^{6}$ cells. Values representing higher viral production after combinatory treatment than after single drug treatment are italicized. Values representing reactivation of the virus observed exclusively after dual treatments are represented in bold. Mean values are represented. "I" indicates below the $300 \mathrm{HIV}-1 \mathrm{RNA}$ copies/ml limit of detection. "NT" indicates not tested conditions. 


\section{DISCUSSION}

A number of chemical compounds have been identified as HIV LRAs. DSF has also been studied and brought forward to clinical trials. ${ }^{9,10}$ In this report, we evaluated reactivation potential of DSF alone or combined with romidepsin in vitro in various latency cell lines of myeloid and T-lymphocytic origins and ex vivo in $\mathrm{CD}^{+}$-depleted PBMCs from $\mathrm{HIV}^{+}$aviremic cART-treated individuals. We observed heterogenous DSF reactivation patterns within myeloid-derived latency cell lines and no reactivation was observed in Jurkat-based T-cell line, highlighting different reactivation patterns between models from different cellular lineage but also between models belonging to the same cellular lineage. Importantly, several recent studies have shown that LRAs have limited reactivation spectra, being effective only in some cell types. For instance, recent work from Baxter et $\mathrm{al}^{22}$ has demonstrated a heterogeneous response of $\mathrm{CD}^{+}{ }^{+}$population to individual LRAs: bryostatin induced the effector memory $\mathrm{CD}^{+} \mathrm{T}$-cell reservoir but had limited effect on the central/transitional memory $\mathrm{CD}^{+}{ }^{+} \mathrm{T}$-cell compartment, contrasting with similar effects observed with ingenol. In addition, it has been recently demonstrated by Chen et $\mathrm{al}^{23}$ that different LRAs reactivate different subsets of latent proviruses. Moreover, we also observed in a previous report a great heterogeneity between patients in terms of reactivation capacity of their ex vivo cell cultures. ${ }^{24}$ Altogether, these recent works and our findings strongly suggest that LRAs may have limited and heterogeneous reactivation spectra in vivo due to cellular and viral heterogeneity of latent reservoirs and that multiple mechanisms may be involved in the reactivation of latent HIV. Previously, Doyon et $\mathrm{al}^{19}$ have investigated the mechanism of DSF action on latent HIV in U1 and Jurkat-based cell lines and have shown that DSF acts through the Akt signaling pathway by decreasing PTEN in U1 cells but not in Jurkat-based cell lines. By contrary, we could not detect PTEN in U1 cells, questioning the role of this factor in DSF-mediated HIV reactivation in this cell line and suggesting a different mechanism. Interestingly, Lin et $\mathrm{al}^{25}$ have shown that DSF treatment results in demethylation of genes hypermethylated in prostate cancer with subsequent reexpression of these genes, suggesting that DSF can act as an epigenetic drug. It remains to be determined whether DSF could act as epigenetic drug on HIV expression.

Remarkably, we could not observe any reactivation effect of DSF ex vivo, in $\mathrm{CD}^{+}$-depleted PBMCs isolated from $\mathrm{HIV}^{+}$aviremic cART-treated individuals. This could be explained at least partially by the fact that most of the HIVinfected cells in blood are $\mathrm{CD}^{+}{ }^{+} \mathrm{T}$ cells with $\mathrm{HIV}$-infected monocytes being extremely low. Although HIV-1 proviral DNA is only present in less than $1 \%$ of circulating monocytes (between $0.01 \%$ and $1 \%$ ), these cells are important viral reservoirs and are responsible for the dissemination of HIV-1 into sanctuaries such as the brain. ${ }^{26}$

Importantly, in our ex vivo studies, we tested 2- and 5$\mu \mathrm{M}$ DSF with the former corresponding to max. plasmatic concentration from the recent dose-escalation clinical trial. ${ }^{10}$ This study showed that even if all doses $(500,1000$, and 2000 $\mathrm{mg}$ ) produced an increase at the level of intracellular HIV
RNA, only the highest dose increased the plasma HIV RNA albeit with very low effect. This effect was extremely modest and an order of magnitude lower than that noted previously with HDACI, romidepsin. ${ }^{13}$ In line with this observation, Mohammadi et $\mathrm{al}^{27}$ have also previously shown in their primary $\mathrm{CD}^{+}{ }^{+} \mathrm{T}$-cell models that DSF treatment successfully increases viral transcription, but fails to effectively enhance viral translation, as the levels of viral-encoded GFP remained low, suggesting the importance of post-transcriptional block(s) as one mechanism leading to HIV latency that needs to be relieved to purge the viral reservoir. It is therefore very likely that DSF may not generate a cellular environment that effectively sustains HIV particle production ex vivo. Similar observation has been reported for SAHA, that is, weak effect at the level of viral particle production in vivo. ${ }^{11}$ Notably, a clear change in plasma HIV-1 RNA with subsequent decrease in the reservoir size has been seen in vivo only for romidepsin (combined with immunotherapy) ${ }^{28}$ and for check point inhibitor nivolumab. ${ }^{29}$ Importantly, no study so far affected time from interruption of cART to viral rebound. These observations and recent studies demonstrating cell-type specificity of LRAs suggest that to achieve high levels of latency reversal, combinations of mechanistically distinct LRAs that exhibit broad spectra of reactivation (being active in multiple cell types) most likely combined with immunotherapy may be required.

In this report, we tested combined treatments of DSF and romidepsin. This LRA combination is of particular clinical interest. DSF has an excellent safety profile given the long history of chronic use for alcohol dependence. Romidepsin has also been evaluated in vivo, and the magnitude of HIV-1 induction after romidepsin administration is greater than anything previously reported for any LRA tested in humans. ${ }^{13} \mathrm{We}$ evaluated simultaneous and sequential combined treatments of those 2 promising LRAs in vitro and ex vivo. Moreover, with the purpose to find the optimal conditions for synergistic studies, we evaluated several concentrations of both LRAs including doses corresponding to maximal plasmatic concentrations, achieved in clinical trials, for both LRAs. Importantly, we observed significant synergistic effects only in U1 cells, and only modest addictive effect was observed ex vivo. Laird et $\mathrm{al}^{21}$ have also observed that DSF+romidepsin combined treatments did not produce synergistic but only modest additive effect in ex vivo cultures of resting $\mathrm{CD}^{+} \mathrm{T}$ cells from infected individuals; however, lower dose of DSF $(0.5 \mu \mathrm{M})$ has been tested in this study. Here, for synergistic ex vivo studies, we evaluated higher doses of DSF, 2 and $5 \mu \mathrm{M}$, with the former corresponding to max. plasmatic concentration from the recent dose-escalation clinical trial.

In summary, cell-type-specific and distinct reactivation patterns of DSF highlight the heterogeneity of HIV reservoirs and that multiple molecular mechanisms contribute to HIV latency that need to be relieved to reach a cure. Notably, demonstration of weak ex vivo reactivation effect of combined DSF+romidepsin treatments should be considered for designing future clinical trials and suggest that to achieve high levels of latency reversal, combination 
of broad-spectrum LRAs (being active in multiple cell types) may be required.

Finally, DSF selectivity for myeloid cells suggests that DSF could represent a good LRA candidate to reactivate this neglected reservoir of myeloid origin including monocytes and microglial cells.

\section{ACKNOWLEDGMENTS}

The authors thank the $H I V-1^{+}$patients for their willingness to participate in this study. The authors thank the nursing team of CHU Saint-Pierre hospital (Brussels, Belgium), Elodie Goudeseune, Joëlle Cailleau, and Annick Caestecker, who cared for the patients. The authors thank Ludivine David and Adeline Melard from Christine Rouzioux's laboratory and Hilde Vereertbrugghen from Francis Corazza's laboratory for excellent technical assistance. The authors thank the transfusion center from Charleroi (Hainaut, Belgium) and principally Jacqueline Pineau for providing the blood from HIV-negative donors.

\section{REFERENCES}

1. Blankson JN, Persaud D, Siliciano RF. The challenge of viral reservoirs in HIV-1 infection. Annu Rev Med. 2002;53:557-593.

2. Crowe SM, Sonza S. HIV-1 can be recovered from a variety of cells including peripheral blood monocytes of patients receiving highly active antiretroviral therapy: a further obstacle to eradication. J Leukoc Biol. 2000;68:345-350.

3. Bergamaschi A, Pancino G. Host hindrance to HIV-1 replication in monocytes and macrophages. Retrovirology. 2010;7:31.

4. Chomont N, El-Far M, Ancuta P, et al. HIV reservoir size and persistence are driven by $\mathrm{T}$ cell survival and homeostatic proliferation. Nat Med. 2009; $15: 893-900$

5. Bouchat S, Delacourt N, Kula A, et al. Sequential treatment with 5-aza2'-deoxycytidine and deacetylase inhibitors reactivates HIV-1. EMBO Mol Med. 2016,8:117-138.

6. Darcis G, Kula A, Bouchat S, et al. An in-depth comparison of latencyreversing agent combinations in various in vitro and ex vivo HIV-1 latency models identified bryostatin-1+JQ1 and ingenol-B+JQ1 to potently reactivate viral gene expression. Plos Pathog. 2015;11: e1005063.

7. Chun TW, Davey RT Jr, Ostrowski M, et al. Relationship between preexisting viral reservoirs and the re-emergence of plasma viremia after discontinuation of highly active anti-retroviral therapy. Nat Med. 2000;6: 757-761.

8. Darcis G, Van Driessche B, Van Lint C. HIV latency: should we shock or lock? Trends Immunol. 2017;38:217-228.

9. Spivak AM, Andrade A, Eisele E, et al. A pilot study assessing the safety and latency-reversing activity of disulfiram in HIV-1-infected adults on antiretroviral therapy. Clin Infect Dis. 2014,58:883-890.

10. Elliott JH, McMahon JH, Chang CC, et al. Short-term administration of disulfiram for reversal of latent HIV infection: a phase 2 dose-escalation study. Lancet HIV. 2015;2:e520-e529.
11. Archin NM, Liberty AL, Kashuba AD, et al. Administration of vorinostat disrupts HIV-1 latency in patients on antiretroviral therapy. Nature. 2012,487:482-485.

12. Rasmussen TA, Tolstrup M, Brinkmann CR, et al. Panobinostat, a histone deacetylase inhibitor, for latent-virus reactivation in HIV-infected patients on suppressive antiretroviral therapy: a phase 1/2, single group, clinical trial. Lancet HIV. 2014;1:e13-e21.

13. Søgaard OS, Graversen ME, Leth S, et al. The depsipeptide romidepsin reverses HIV-1 latency in vivo. PLoS Pathog. 2015;11:e1005142.

14. Kulkosky J, Culnan DM, Roman J, et al. Prostratin: activation of latent HIV-1 expression suggests a potential inductive adjuvant therapy for HAART. Blood. 2001,98:3006-3015.

15. Abreu CM, Price SL, Shirk EN, et al. Dual role of novel ingenol derivatives from Euphorbia tirucalli in HIV replication: inhibition of de novo infection and activation of viral LTR. PLoS One. 2014;9:e97257.

16. Bouchat S, Gatot JS, Kabeya K, et al. Histone methyltransferase inhibitors induce HIV-1 recovery in resting CD4(+) T cells from HIV1-infected HAART-treated patients. AIDS. 2012;26:1473-1482.

17. Tsai A, Irrinki A, Kaur J, et al. Toll-like receptor 7 agonist GS-9620 induces HIV expression and HIV-specific immunity in cells from HIVinfected individuals on suppressive antiretroviral therapy. $J$ Virol. 2017; 91:e02166-e 021616

18. Faiman MD, Jensen JC, Lacoursiere RB. Elimination kinetics of disulfiram in alcoholics after single and repeated doses. Clin Pharmacol Ther. 1984;36:520-526.

19. Doyon G, Zerbato J, Mellors JW, et al. Disulfiram reactivates latent HIV1 expression through depletion of the phosphatase and tensin homolog. AIDS. 2013;27:F7-F11.

20. Xing S, Bullen CK, Shroff NS, et al. Disulfiram reactivates latent HIV-1 in a Bcl-2-transduced primary CD4+ $\mathrm{T}$ cell model without inducing global T cell activation. $J$ Virol. 2011;85:6060-6064.

21. Laird GM, Bullen CK, Rosenbloom DI, et al. Ex vivo analysis identifies effective HIV-1 latency-reversing drug combinations. J Clin Invest. 2015,125:1901-1912.

22. Baxter AE, Niessl J, Fromentin R, et al. Single-cell characterization of viral translation-competent reservoirs in HIV-infected individuals. Cell Host Microbe. 2016,20:368-380.

23. Chen HC, Martinez JP, Zorita E, et al. Position effects influence HIV latency reversal. Nat Struct Mol Biol. 2017;24:47-54.

24. Darcis G, Bouchat $S$, Kula A, et al. Reactivation capacity by latencyreversing agents ex vivo correlates with the size of the HIV-1 reservoir AIDS. 2016

25. Lin J, Haffner MC, Zhang Y, et al. Disulfiram is a DNA demethylating agent and inhibits prostate cancer cell growth. Prostate. 2011;71:333343.

26. Le Douce V, Herbein G, Rohr O, et al. Molecular mechanisms of HIV-1 persistence in the monocyte-macrophage lineage. Retrovirology. 2010;7: 32.

27. Mohammadi P, di Iulio J, Munoz M, et al. Dynamics of HIV latency and reactivation in a primary CD4+ T cell model. PLoS Pathog. 2014;10: e1004156.

28. Leth S, Schleimann MH, Nissen SK, et al. Combined effect of Vacc-4x, recombinant human granulocyte macrophage colony-stimulating factor vaccination, and romidepsin on the HIV-1 reservoir (REDUC): a singlearm, phase 1B/2A trial. Lancet HIV. 2016;3:e463-e472.

29. Guihot A, Marcelin AG, Massiani MA, et al. Drastic decrease of the HIV reservoir in a patient treated with nivolumab for lung cancer. Ann Oncol. 2017. 